\title{
Deforming black holes with odd multipolar differential rotation boundary
}

\author{
Shuo Sun, ${ }^{*}$ Tong-Tong $\mathrm{Hu},{ }^{\dagger}$ Hong-Bo Li, ${ }^{*}$ and Yong-Qiang Wang $\oplus^{\S}$ \\ Research Center of Gravitation, Institute of Theoretical Physics, Key Laboratory for Magnetism \\ and Magnetic of the Ministry of Education, Lanzhou University, Lanzhou 730000, China
}

(Received 22 July 2019; published 28 October 2019)

\begin{abstract}
Motivated by the novel asymptotically global $\mathrm{AdS}_{4}$ solutions with deforming horizon in [J. Markeviciute and J. E. Santos, Stirring a black hole, J. High Energy Phys. 02 (2018) 060.], we analyze the boundary metric with odd multipolar differential rotation and numerically construct a family of deforming solutions with tripolar differential rotation boundary, including two classes of solutions: solitons and black holes. We find that the maximal values of the rotation parameter $\varepsilon$, below which the stable large black hole solutions could exist, are not a constant for $T>T_{\text {schw }}=\sqrt{3} / 2 \pi \simeq 0.2757$. When temperature is much higher than $T_{\text {schw }}$, even though the norm of Killing vector $\partial_{t}$ keeps timelike for some regions of $\varepsilon<2$, solitons and black holes with tripolar differential rotation could be unstable and develop hair due to superradiance. As the temperature $T$ drops toward $T_{\text {schw }}$, we find that though there exists the spacelike Killing vector $\partial_{t}$ for some regions of $\varepsilon>2$, solitons and black holes still exist and do not develop hair due to superradiance. Moreover, for $T \leq T_{\text {schw }}$, the curves of entropy first combine into one curve and then separate into two curves again; in the case of each curve there are two solutions at a fixed value of $\varepsilon$. In addition, we study the deformations of horizon for black holes by using an isometric embedding in the hyperbolic threedimensional space. Furthermore, we also study the quasinormal modes of the solitons and black holes, which have analogous behaviors to that of dipolar rotation and quadrupolar rotation.
\end{abstract}

DOI: 10.1103/PhysRevD.100.084063

\section{INTRODUCTION}

The uniqueness theorem of black holes [1-4] in classical general relativity has shown that the asymptotically flat black hole solutions with zero angular momentum in four dimensions are named as Schwarzschild black holes, which have a spherical event horizon. It is well known that in fourdimensional anti-de Sitter (AdS) spacetime, there exist some solutions with noncompact planar, hyperbolic horizons and compact horizons of arbitrary genus $g$. Recently, the asymptotically AdS black holes in the context of antide Sitter/comformal field theory (AdS/CFT) correspondence [5-7] have aroused great interest, and it is especially important to study the physical properties and applications of such black holes.

Because the asymptotically AdS black hole has a conformal boundary at infinity, we could deform the

\footnotetext{
*sunsh17@lzu.edu.cn

†hutt17@1zu.edu.cn

"lihb2017@lzu.edu.cn

${ }^{\S}$ Corresponding author. yqwang@lzu.edu.cn
}

Published by the American Physical Society under the terms of the Creative Commons Attribution 4.0 International license. Further distribution of this work must maintain attribution to the author(s) and the published article's title, journal citation, and DOI. Funded by SCOAP. boundary metric. By solving the Einstein equation, we could obtain the solution of black hole with deforming horizon, which means that the curvature of the horizon is not a constant and the horizon could have various deformations. There are two methods to get the solutions of deforming black holes; one method is analytically constructing a family of the hyperbolic AdS black holes [8] with deforming horizon in four-dimensional spacetime by using the AdS C-metric [9-11]. In addition, a class of fourdimensional AdS black holes with noncompact event horizons of finite area was found in [12,13], and black holes with bottle-shaped horizons were found in [14]. Another method is to study the deforming black holes by using numerical methods.

It is important to study the stability of the black hole solutions. Recently, lots of work has pointed out that the instability of black hole solutions could be related to superradiance. The super-radiance in the asymptotically flat context was studied in [15-22], and the case of rotating black holes in AdS spacetimes was studied in [23-26]. The idea of super-radiance instability is that if the angular velocity $\Omega_{H}$ of the black hole in AdS spacetime is large enough, this black hole will have a region called the ergoregion. When waves with azimuthal quantum number $m$ and frequency $0<\omega<m \Omega_{H}$ are scattered, the ergoregion could store negative energy, and a rotating black hole with this ergoregion becomes unstable. In [27], authors found 
that the ergoregion was the region where the Killing vector became spacelike, and in this region, both solitons and black holes would develop hair due to super-radiance.

In [28], the author constructed a family of deforming solutions with differential rotation boundary, including the solitons and black holes. These solutions have nontrivial boundary metrics with a dipoalr differential rotation profile,

$$
\Omega(\theta)=\varepsilon \cos (\theta),
$$

where the parameter $\varepsilon$ is the boundary rotation parameter and the polar angle $\theta$ is restricted to the interval $(0, \pi)$. The rotation profile $\Omega(\theta)$ is antisymmetric on the equatorial plane $\theta=\pi / 2$. When the rotation boundary parameter $\varepsilon$ is larger than a critical value $\varepsilon=2$, the Killing vector $\partial_{t}$ becomes spacelike for certain regions of $\theta$ with $\varepsilon>2$, which are so-called ergoregions. For $\varepsilon>2$, both solitons and black holes develop hair due to super-radiance, and the spacetime with ergoregions in AdS may be unstable due to the super-radiant scattering [27]. Furthermore, a family of deforming vacuum solutions with a noncompact, differential rotation boundary metric was numerically studied in [29]. In [30], authors studied the influence of the hyperbolic and compact AdS black holes when the boundary metric changes by using the AdS C-metric. One could construct the black holes with deforming horizon in $D=5$ minimal gauged supergravity by introducing the matter fields [31].

We are interested in whether there are deforming solutions with the multipolar differential rotation boundary. In [32] authors try to numerically solve the Einstein equations and give a family of deforming solitons and black holes with even multipolar differential rotation boundary, which have the antisymmetric rotation profile with respect to reflections on the equatorial plane and the total angular momentum of black hole is 0 . We consider the configuration of quadrupolar rotation boundary, in particular, and we obtain the numerical results of the deforming solitons and black holes. In quadrupolar differential rotation situation, solitons and black holes do not develop hair due to super-radiance when the norm of the Killing vector $\partial_{t}$ becomes spacelike for certain regions of $\theta$ with $\varepsilon \in$ $(2,2.281)$. This is very different from the dipolar differential rotation situation. It could be observed that the black hole horizon is deformed into four hourglass shapes by isometric embedding in the horizon. Furthermore, the numerical solutions of entropy and the quasinormal modes have analogous properties to that of dipolar differential rotation boundary [28].

In this paper, we attempt to numerically solve the Einstein equation and give a family of deforming solitons and black holes with odd multipolar differential rotation boundary, which has the symmetric rotation profile with respect to reflections on the equatorial plane. Especially, considering the configuration of tripolar differential rotation boundary, we obtain the numerical results of the deforming solitons and black holes. Comparing with the results of dipolar differential rotation, we find that the norm of Killing vector $\partial_{t}$ becomes spacelike for certain regions of $\theta$ with $\varepsilon \in(2,2.124)$ at the temperature $T=1 / \pi$. However, solitons and black holes with tripolar differential rotation do not develop hair due to super-radiance, which is different from the case of dipolar rotation. Furthermore, we study the numerical solutions of entropy, and it is different from the cases of dipolar rotation and quadrupolar rotation when temperature increased; the maximal values of rotation parameter $\varepsilon$, below which the stable large black hole solutions could exist, are not a constant. When temperature is much higher than $T_{\text {schw }}=\sqrt{3} / 2 \pi \simeq 0.2757$, for which $T_{\text {Schw }}$ is the minimal temperature of the $\mathrm{AdS}_{4}$-Schwarzschild black hole, the norm of Killing vector $\partial_{t}$ keeps timelike for some regions of $\varepsilon<2$; solitons and black holes could be unstable and develop hair due to super-radiance. We find that the curves of entropy have different behaviors at different temperatures. When $T>T_{\text {Schw }}$, we first observe that the curves of entropy for large and small branches of black hole solutions separate into two curves. Then two branches of curves of entropy combine into one curve when $T=T_{\text {Schw }}$. When $T<T_{\text {Schw }}$, we again observe that the two curves of entropy separate into two curves, and each curve of entropy has two solutions at a fixed value of $\varepsilon$. In addition, by isometric embedding the horizon into hyperbolic space, the black hole horizon is deformed into three protrusions. At last we study the quasinormal modes, which have the analogous properties to that of dipolar and quadrupolar rotation boundary in [28,32].

The paper is organized as follows. In Sec. II, we introduce the model of the deforming black holes with odd multipolar differential rotation boundary and the numerical DeTurck method. In Sec. III, we construct numerical models of soliton solutions with tripolar differential rotation boundary and show the numerical results of Kretschmann scalar and quasinormal modes. The numerical models and results of deforming black holes with tripoalr differential rotation boundary are shown in Sec. IV. The conclusions and discussions are given in the last section.

\section{MODEL AND NUMERICAL METHOD}

We first begin with the model of the four-dimensional Einstein-Hilbert action with a negative cosmological constant $\Lambda$,

$$
S=\frac{1}{16 \pi G} \int d^{4} x \sqrt{-g}(R-2 \Lambda),
$$

where $G$ is the gravitational constant, and the cosmological constant $\Lambda$ could be represented by the AdS radius $L$ as $\Lambda=-3 / L^{2}, g$ is the determinant of the metric tensor, and $R$ is Ricci scalar. The equation of motion derived from (2.1) takes the following form, 


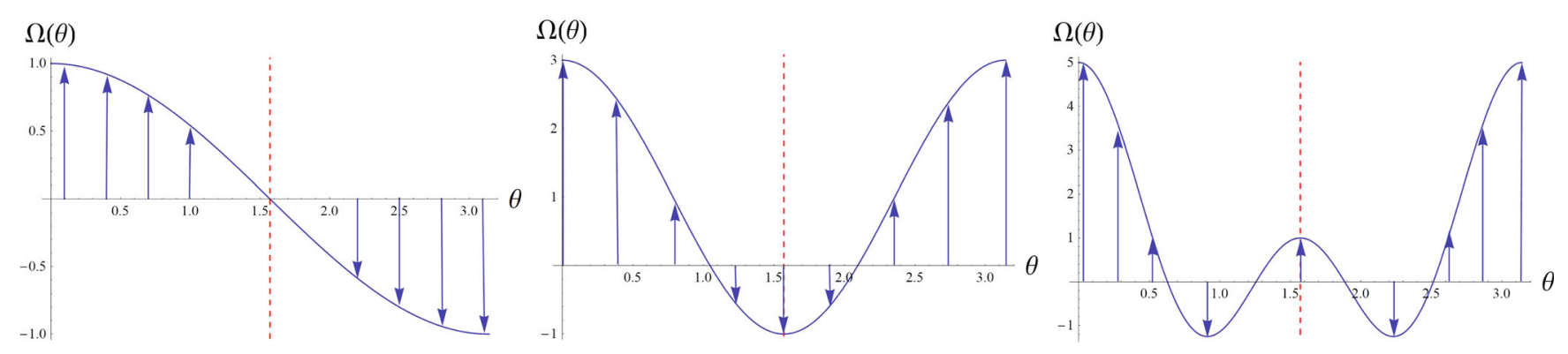

FIG. 1. The differential rotation profile $\Omega$ as a function of $\theta$ with dipolar rotation (left panel), tripolar rotation (middle panel), and pentapolar rotation (right panel), respectively. In all three graphs the arrow lines denote the orientation of differential rotation and the red dashed lines mean $\theta=\pi / 2$.

$$
G_{a b} \equiv R_{a b}+\frac{3}{L^{2}} g_{a b}=0
$$

The solution of Einstein equation (2.2) is the well-known AdS-Schwarzschild black hole that described the static spherically symmetric black hole with mass. The metric is given by

$$
\begin{aligned}
d s^{2}= & -\left(1-\frac{2 M}{r}+\frac{r^{2}}{L^{2}}\right) d t^{2} \\
& +\left(1-\frac{2 M}{r}+\frac{r^{2}}{L^{2}}\right)^{-1} d r^{2}+r^{2} d \Omega^{2},
\end{aligned}
$$

where $d \Omega^{2}$ is the metric on the sphere $S^{2}$. The constant $M$ is the mass of black hole as measured from the infinite boundary. The horizon radius, denoted by $r_{+}$, is the largest root of equation

$$
1-\frac{2 M}{r}+\frac{r^{2}}{L^{2}}=0
$$

The Hawking temperature $T_{H}$ of the AdS-Schwarzschild black hole is given by

$$
T_{H}=\frac{L^{2}+3 r_{+}^{2}}{4 \pi L^{2} r_{+}} .
$$

As it is near the infinity, the metric (2.3) is asymptotic to the anti-de Sitter spacetime, and the conformal boundary metric is given by

$$
d s_{\partial}^{2}=r^{2}\left(-d t^{2}+d \theta^{2}+\sin ^{2} \theta d \phi^{2}\right) .
$$

In [28], the authors added differential rotation to the boundary metric to obtain the new asymptotic anti-de Sitter solution, which is given by

$$
d s_{\partial}^{2}=r^{2}\left(-d t^{2}+d \theta^{2}+\sin ^{2} \theta[d \phi+\Omega(\theta) d t]^{2}\right),
$$

where $\Omega(\theta)=\varepsilon \cos \theta$ is the dipolar differential rotation. The norm of Killing vector $\partial_{t}$ is

$$
\left\|\partial_{t}\right\|^{2}=-1+\frac{\varepsilon^{2}}{4} \sin ^{2}(2 \theta)
$$

and the maximum value is at $\theta=\pi / 4$.

In order to construct higher odd multipolar differential rotation of the conformal boundary, we could take the following form of Killing vector $\partial_{t}$,

$$
\left\|\partial_{t}\right\|^{2}=-1+\frac{\varepsilon^{2}}{4} \sin ^{2}(k \theta), \quad k=3,5,7, \ldots,
$$

which corresponds to the odd multipole differential rotation,

$$
\Omega(\theta)= \begin{cases}2 \varepsilon[1+2 \cos (2 \theta)], & k=3, \\ 2 \varepsilon[\csc (\theta) \sin (5 \theta)], & k=5, \\ 2 \varepsilon[\csc (\theta) \sin (7 \theta)], & k=7, \\ \ldots, & k=\cdots\end{cases}
$$

For the case of $k=3,\left\|\partial_{t}\right\|^{2}$ has maximal value at $\theta=\pi / 6$, and when $\varepsilon>2$ in the certain regions of $\theta$, the Killing vector $\partial_{t}$ becomes spacelike. In Fig. 1, we draw the graphs of differential rotation $\Omega$ as the functions of $\theta$ with dipolar rotation (left panel), tripolar rotation (middle panel), and pentapolar rotation (right panel), respectively. In all three graphs the arrow lines denote the orientation of differential rotation. The dipolar differential rotation $\Omega$ is the antisymmetric function with respect to reflections on the equatorial plane $\theta=\pi / 2$, and the total angular momentum of the black hole is 0 . But in the odd polar situation, $\Omega$ is the symmetric function with respect to reflections on the equatorial plane $\theta=\pi / 2$.

In order to obtain the numerical solution of Einstein equation (2.2), we use the DeTurck method [33-35]. The DeTurck method is to add a gauge fixing term to the Einstein equation and keeps the equations in elliptic form. This elliptic set of equations is known as Einstein-DeTurck equations,

$$
R_{a b}+\frac{3}{L^{2}} g_{a b}-\nabla_{(a} \xi_{b)}=0,
$$


where $\xi^{a}=g^{b c}\left(\Gamma_{b c}^{a}[g]-\Gamma_{b c}^{a}[\tilde{g}]\right)$ is the DeTurck vector, and $\Gamma_{b c}^{a}[\tilde{g}]$ is the Levi-Civita connection for the appropriate reference metric $\tilde{g}$. For the case of solitons, we require the reference metric to approach the pure $\mathrm{AdS}_{4}$ with a rotating conformal boundary metric in global coordinates. Similarly, for the case of black holes, we require the reference metric to approach the $\mathrm{AdS}_{4}$-Schwarzchild black holes with a rotating conformal boundary metric in global coordinates. In addition, if the gauge choice $\xi^{a}=0$, the solution of equation (2.11) is equal to the solution of the Einstein equation.

By using numerical methods to solve these equations of motion, we obtain two classes of solutions, which are horizonless soliton solutions with $r_{H}=0$ and black hole solutions with $r_{H}>0$. The soliton solutions could be seen as deformations of the global anti-de Sitter spacetime, and the black hole solutions correspond to deformations of $\mathrm{AdS}_{4}$ black holes. For simplicity, in our paper we only show the numerical results of the tripolar differential rotation $(k=3)$, and the situations of higher odd-multipole differential rotation have similar behaviors as that of solutions of the tripolar differential rotation.

\section{SOLITON SOLUTIONS}

We make a coordinate transformation that the variable $y$ is related to the $\mathrm{AdS}_{4}$ radial coordinate via $r=L y \sqrt{2-y^{2}}$ / $\left(1-y^{2}\right)$, and $x$ is the variable about the standard polar angle on $S^{2}$ by $\sin \theta=1-x^{2}$, which implies that the new radial coordinate $y \in[0,1]$ and polar angle coordinate $x \in[0,1]$. Thus the inner and outer boundaries of the shell are fixed at $y=0$ and $y=1$, respectively. In order to obtain the numerical solutions with a tripolar differential rotation (2.10), we introduce the following ansatz of solitonic solutions,

$$
\begin{aligned}
d s^{2}= & \frac{L^{2}}{\left(1-y^{2}\right)^{2}}\left\{-U_{1} d t^{2}+\frac{4 U_{2}}{2-y^{2}} d y^{2}\right. \\
& +y^{2}\left(2-y^{2}\right)\left[\frac{4 U_{3}}{2-x^{2}}\left(d x+\frac{1-8 x^{2}+4 x^{4}}{y} U_{4} d y\right)^{2}\right. \\
& \left.\left.+\left(1-x^{2}\right)^{2} U_{5}\left(d \phi+y\left(1-8 x^{2}+4 x^{4}\right) U_{6} d t\right)^{2}\right]\right\},
\end{aligned}
$$

where $U_{i}(i=1, \ldots, 6)$ are the functions of $x$ and $y$. We introduce four functions $U_{i}(i=1,2,3,5)$ in ansatz (3.1), and these four functions correspond to four diagonal terms in the metric. In addition, considering the symmetry of rotation, we introduce $U_{6}$ into the coefficient of the $d t d \phi$ term of the metric. Moreover, the DeTurck method need to introduce $U_{4}$ into the coefficient of the $d x d y$ term of the metric to keep the number of equations of motion consistent with the number of functions $U_{i}(i=1, \ldots, 6)$. Furthermore, when $U_{1}=U_{2}=U_{3}=U_{5}=1$, and $U_{4}=$ $U_{6}=0$, we require that the ansatz could reduce to pure $\mathrm{AdS}_{4}$ in global coordinates.
The tripoalr differential rotation profile $\Omega(\theta) \sim 2 \varepsilon[1+$ $2 \cos (2 \theta)]$ could be written as $1-8 x^{2}+4 x^{4}$ in $(x, y)$ coordinates. In $x \in[0,1]$, the zero value of $\Omega(\theta)$ appears at $x=1 / 2(-1+\sqrt{3})$ and can cause difficulties in numerical calculation. So we introduce new functions $\tilde{U}_{4}=(1-$ $\left.8 x^{2}+4 x^{4}\right) U_{4}$ and $\tilde{U}_{6}=\left(1-8 x^{2}+4 x^{4}\right) U_{6}$; the ansatz (3.1) could be rewritten as

$$
\begin{aligned}
d s^{2}= & \frac{L^{2}}{\left(1-y^{2}\right)^{2}}\left\{-U_{1} d t^{2}+\frac{4 U_{2}}{2-y^{2}} d y^{2}+y^{2}\left(2-y^{2}\right)\right. \\
& \times\left[\frac{4 U_{3}}{2-x^{2}}\left(d x+\frac{1}{y} \tilde{U}_{4} d y\right)^{2}\right. \\
& \left.\left.+\left(1-x^{2}\right)^{2} U_{5}\left(d \phi+y \tilde{U}_{6} d t\right)^{2}\right]\right\},
\end{aligned}
$$

where $U_{i},(i=1,2,3,5)$ and $\tilde{U}_{4}, \tilde{U}_{6}$ are the functions of $x$ and $y$.

We need to obtain the asymptotic behaviors of functions $U_{i}(i=1,2,3,5)$ and $\tilde{U}_{4}, \tilde{U}_{6}$ before numerically solving the partial differential equations, which are also the boundary conditions we need. Because the solutions have properties of polar angle reflection symmetry $\theta \rightarrow \pi-\theta$ on the equatorial plane, we could consider the coordinate range $\theta \in[0, \pi / 2]$, i.e., $x \in[0,1]$. Therefore, we require the functions to satisfy the following Neumann boundary conditions on the equatorial plane $x=0$,

$$
\begin{cases}\partial_{x} U_{i}(0, y)=0, & i=1,2,3,5 \\ \partial_{x} \tilde{U}_{i}(0, y)=0, & i=4,6\end{cases}
$$

In addition, expanding the equations of motion near $x=1$, as a power series in $1-x$, we obtain the condition $U_{3}(1, y)=U_{5}(1, y)$. The boundary condition of $\tilde{U}_{4}$ at $x=1$ is

$$
\tilde{U}_{4}(1, y)=0
$$

and the Neumann boundary conditions on the other functions

$$
\begin{aligned}
\partial_{x} U_{1}(1, y) & =\partial_{x} U_{2}(1, y)=\partial_{x} U_{3}(1, y)=\partial_{x} U_{5}(1, y) \\
& =\partial_{x} \tilde{U}_{6}(1, y)=0 .
\end{aligned}
$$

Moreover, the asymptotic behaviors near the conformal boundary $y=1$ are

$$
\begin{aligned}
& U_{1}(x, 1)=U_{2}(x, 1)=U_{3}(x, 1)=U_{5}(x, 1)=1, \\
& \tilde{U}_{4}(x, 1)=0, \quad \tilde{U}_{6}(x, 1)=\left(1-8 x^{2}+4 x^{4}\right) \varepsilon .
\end{aligned}
$$

Finally, by expanding the equations of motion near $y=0$ as a power series in $y$, we have 

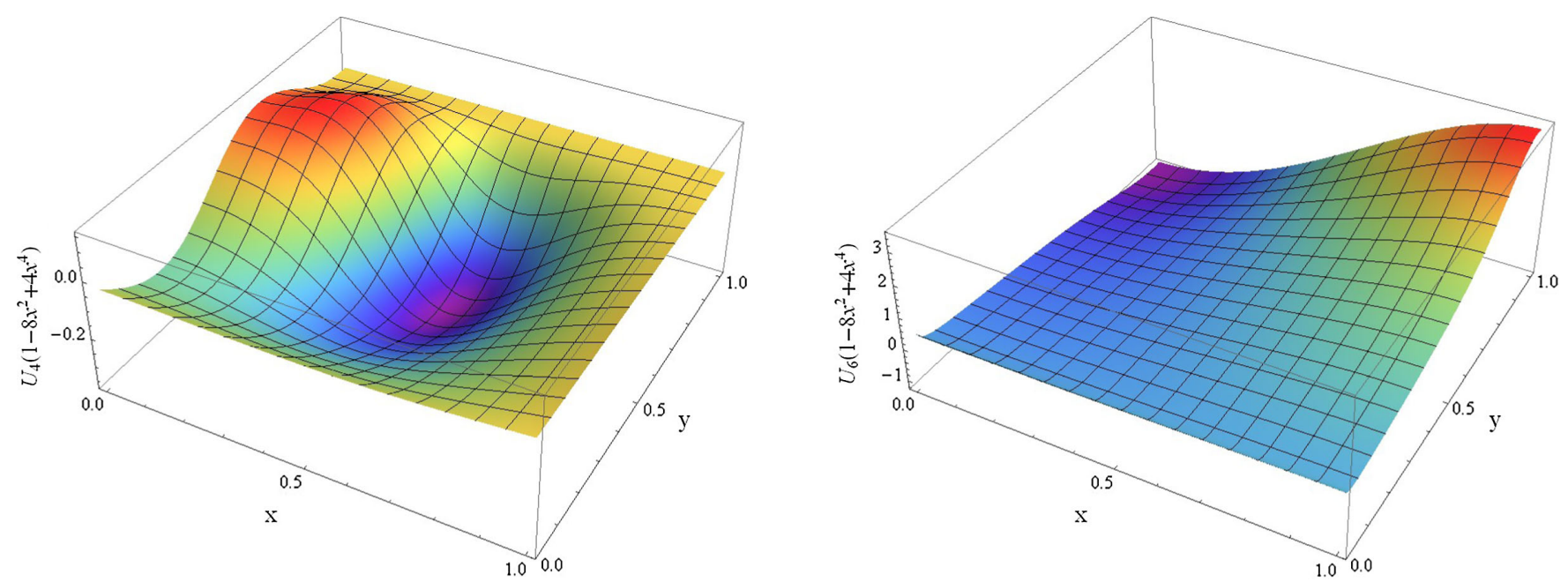

FIG. 2. The left panel shows $U_{4}\left(1-8 x^{2}+4 x^{4}\right)$ and the right panel shows $U_{6}\left(1-8 x^{2}+4 x^{4}\right)$ of the soliton solution with the tripolar boundary rotation. Two solutions have $\varepsilon=2.3$.

$$
\begin{cases}\partial_{y} U_{i}(x, 0)=0, & i=1,2,3,5, \\ \partial_{y} \tilde{U}_{i}(x, 0)=0, & i=4,6 .\end{cases}
$$

We can choose the reference metric $\tilde{g}$ that is used in the DeTurck method with $U_{1}=U_{2}=U_{3}=U_{5}=1, \tilde{U}_{4}=0$, and $\tilde{U}_{6}=\varepsilon\left(1-8 x^{2}+4 x^{4}\right)$.

In Fig. 2, we show the typical soliton results of our numerical code for $\tilde{U}_{4}$ in the left panel and $\tilde{U}_{6}$ in the right panel with tripolar boundary rotation, and the two figures have the same parameter $\varepsilon=2.3$. According to the numerical results, we find that there exist stationary axisymmetric soliton solutions for $\varepsilon<\varepsilon_{c}=2.5264$, where $\varepsilon_{c}$ is the maximal value.

We use the finite element methods in the integration regions $0 \leq x \leq 1$ and $0 \leq y \leq 1$ defined on nonuniform grids, allowing the grids to be more fine grid points near the boundaries of $y=0$ and $y=1$. Our iterative process is the Newton-Raphson method. The relative error for the numerical solutions in this work is estimated to be below $10^{-6}$. In order to keep good agreement with the aforementioned error, the grid size has to be increased and typically a $120 \times 200$ to $120 \times 370$ grid was used. In all the calculations and plots, we set $L=1$.

\section{A. Kretschman scalar}

It is very important to know whether the spacetime of the solution is regular or not when we obtain a solution of the Einstein equation. In general, the Ricci scalar is the simplest curvature invariant of a Riemannian manifold. However, in our model the Ricci tensor is $R=2 \Lambda$. The other choice is to check the behavior of the Kretschmann scalar, which is invariant, and it could indicate the flatness of a chosen manifold. The Kretschmann scalar is written as

$$
K=R_{\alpha \beta \gamma \delta} R^{\alpha \beta \gamma \delta}
$$

where $R_{\alpha \beta \gamma \delta}$ is the Riemann curvature tensor and because it is a sum of squares of tensor components, Kretschmann scalar is a quadratic invariant.

We present the maximal value of the Kretschmann scalar as the function of $\varepsilon$ in Fig. 3; it could be seen that the maximal value of the Kretschmann scalar increases with the increase of $\varepsilon$. When the rotation parameter $\varepsilon$ approaches 0 , the maximal value of $K$ is equal to the maximal value of $K$ for pure $\mathrm{AdS}_{4}$. Furthermore, for $\varepsilon \in\left(2.5204, \varepsilon_{c}\right)$, we find that there are two soliton solutions existing for a fixed value of $\varepsilon$, but it is too difficult for our numerical method to calculate the maximal value for the second branch of Kretschmann scalar $K$ when $\varepsilon<2.5204$.

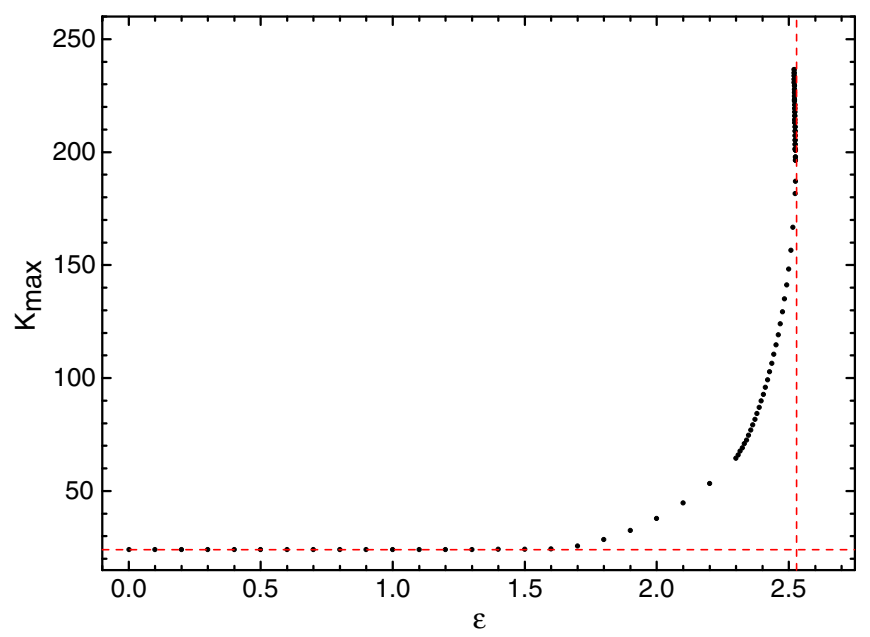

FIG. 3. The maximum of the Kretschmann scalar as a function of the boundary rotation parameter $\varepsilon$ for the soliton solutions. The vertical red dashed line marks the max $K=24 / L^{2}$ for $\mathrm{AdS}_{4}$ spacetime, and the horizontal red dashed line marks $\varepsilon_{c}=2.5264$. 


\section{B. The quasinormal modes}

In order to study the linear stability of soliton solutions with tripolar boundary rotation, we study the quasinormal modes, which are characteristics of the background spacetime. Following the method in papers $[25,28,36]$, we consider a free, massless scalar field, obeying a massless Klein-Gordon equation

$$
\nabla^{2} \Psi=0
$$

and the scalar field could be separated into the standard form

$$
\Psi=\hat{\Psi}_{\omega, m}(x, y) e^{-i \omega t+i m \psi}, \quad m= \pm 1, \pm 2, \ldots,
$$

where $\omega$ is the frequency of the complex scalar field and $m$ is the azimuthal harmonic index. On the soliton background (3.2), the scalar field could be separated into

$\Psi(t, x, y, \psi)=e^{i(m \psi-\omega t)} y^{|m|}\left(1-y^{2}\right)^{3}\left(1-x^{2}\right)^{|m|} \xi(x, y)$.

The boundary conditions are imposed as follows: at $x= \pm 1$, we require $\partial_{x} \xi(x, y)=0$; at $y=0$, we require $\partial_{y} \xi(x, y)=0$, and at $y=1$, we require

$$
\partial_{y} \xi(x, y)=-|m| \xi(x, y) .
$$

In Fig. 4, we plot the normal mode frequencies $\omega$ as the functions of the rotation parameter $\varepsilon$ for corresponding values of $m$. We could see that the frequency $\omega$ became negative at a specific value of $\varepsilon$ when $m=24$, and the frequencies are always positive modes in the spectrum of perturbations when $m \leq 23$. When $\varepsilon$ is large enough, the

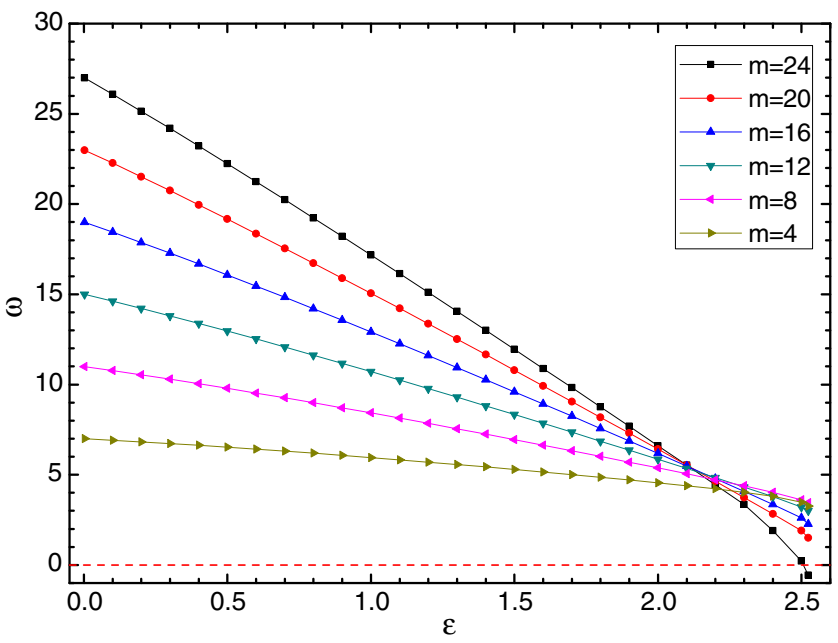

FIG. 4. Normal mode frequencies against the rotation parameter $\varepsilon$ for the soliton metric. The red dashed line shows $\omega=0$. solutions of the soliton become unstable because of superradiance, and the negative frequency appears. These negative frequencies correspond to the unstable modes, which means that the scalar hair condensation could be formed. We can see from Fig. 4, when $m \geq 24$, that one can expect that some branches of soliton with scalar hair $\Psi$ condensation could be found.

\section{BLACK HOLE SOLUTIONS}

In order to obtain the black hole solutions with a tripolar differential rotation (2.10), we introduce the following ansatz,

$$
\begin{aligned}
d s^{2}= & \frac{L^{2}}{\left(1-y^{2}\right)^{2}}\left\{-y^{2} \tilde{\Gamma}(y) U_{1} d t^{2}+\frac{4 y_{p}^{2} U_{2} d y^{2}}{\tilde{\Gamma}(y)}\right. \\
& +y_{p}^{2}\left[\frac{4 U_{3}}{2-x^{2}}\left(d x+\left(1-8 x^{2}+4 x^{4}\right) y U_{4} d y\right)^{2}\right. \\
& \left.\left.+\left(1-x^{2}\right)^{2} U_{5}\left(d \phi+y^{2}\left(1-8 x^{2}+4 x^{4}\right) U_{6} d t\right)^{2}\right]\right\},
\end{aligned}
$$

where

$$
\begin{aligned}
& \tilde{\Gamma}(y)=\Gamma(y) \sigma+y_{p}^{2}(1-\sigma), \\
& \Gamma(y)=\left(1-y^{2}\right)^{2}+y_{p}^{2}\left(3-3 y^{2}+y^{4}\right),
\end{aligned}
$$

where $U_{i}(i=1, \ldots, 6)$ are the functions of $x$ and $y$, and the parameters $\left(y_{p}, \sigma\right)$ are related to the black hole temperature. The variable $y$ is related to the usual radial coordinate via $r=L y_{p} /\left(1-y^{2}\right)$, and $x$ is the variable about standard polar angle on $S^{2}$ via $\sin \theta=1-x^{2}$. Similar to the case of the soliton, we introduce the functions $U_{i}(i=1, \ldots, 6)$ in the ansatz. When $U_{1}=U_{2}=U_{3}=U_{5}=\sigma=1, U_{4}=$ $U_{6}=0$, we require the ansatz to reduce to the $\mathrm{AdS}_{4}$ Schwarzchild black hole in global coordinates.

Considering the same difficulties in numerical calculation, which are similar to the case of the soliton, we obtain the ansatz with $\tilde{U}_{4}=U_{4}\left(1-8 x^{2}+4 x^{4}\right)$ and $\tilde{U}_{6}=U_{6}(1-$ $\left.8 x^{2}+4 x^{4}\right)$ as follows,

$$
\begin{aligned}
d s^{2}= & \frac{L^{2}}{\left(1-y^{2}\right)^{2}}\left\{-y^{2} \tilde{\Gamma}(y) U_{1} d t^{2}+\frac{4 y_{p}^{2} U_{2} d y^{2}}{\tilde{\Gamma}(y)}\right. \\
& +y_{p}^{2}\left[\frac{4 U_{3}}{2-x^{2}}\left(d x+y \tilde{U}_{4} d y\right)^{2}\right. \\
& \left.\left.+\left(1-x^{2}\right)^{2} U_{5}\left(d \phi+y^{2} \tilde{U}_{6} d t\right)^{2}\right]\right\}
\end{aligned}
$$

where $U_{i}(i=1,2,3,5)$ and $\tilde{U}_{4}, \tilde{U}_{6}$ are the functions of $x$ and $y$.

The Hawking temperature is computed in the usual way by metric, 

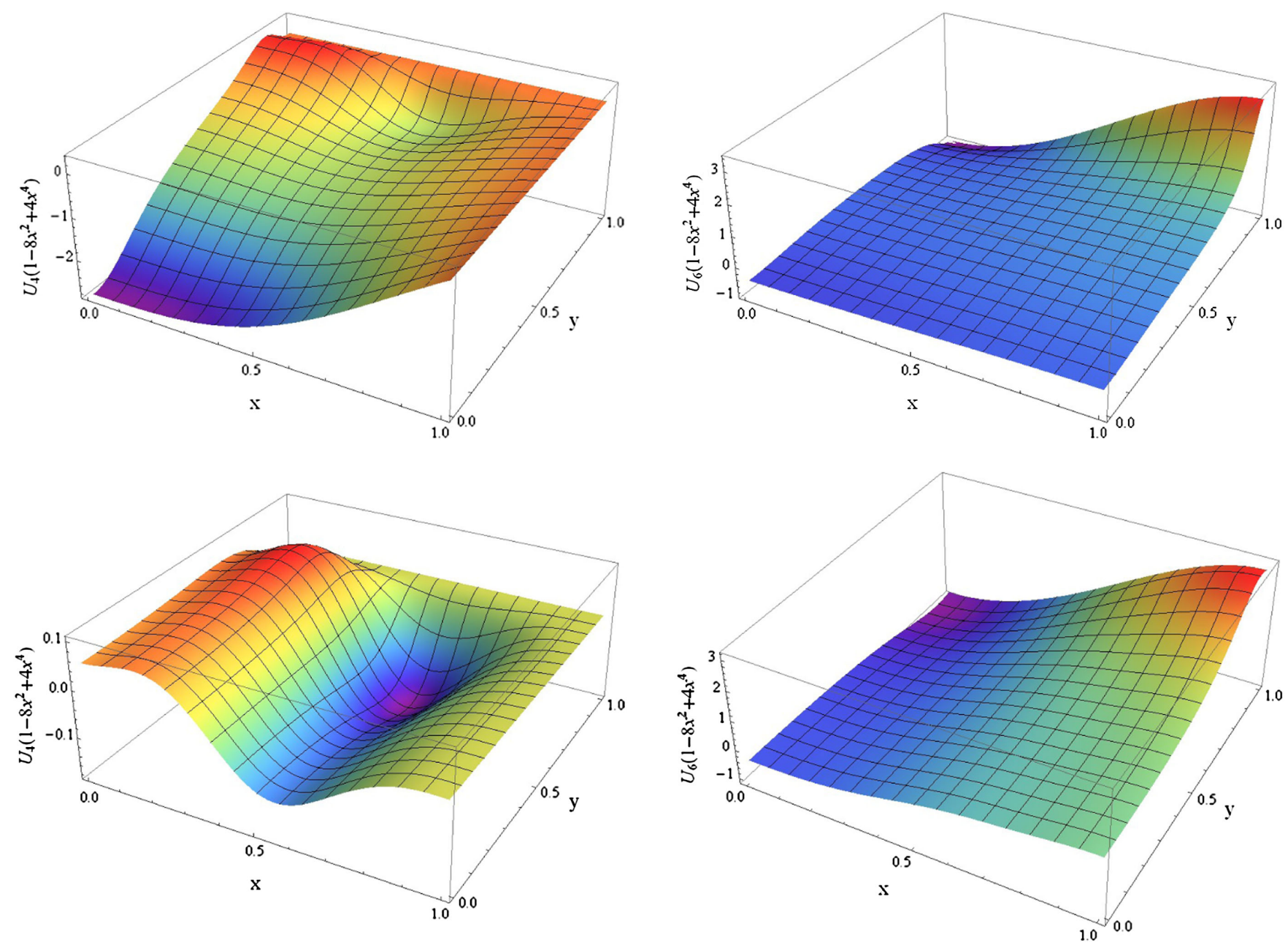

FIG. 5. Top: The distributions of $\tilde{U}_{4}$ and $\tilde{U}_{6}$ as functions of $x$ and $y$ for a small black hole. Bottom: The distributions of $\tilde{U}_{4}$ and $\tilde{U}_{6}$ as functions of $x$ and $y$ for a large black hole. All the solutions have the rotation parameter $\varepsilon=2$.

$T=\left.\frac{1}{4 \pi} \sqrt{-g^{t t} g^{\alpha \beta} \partial_{\alpha} g_{t t} \partial_{\beta} g_{t t}}\right|_{r=r_{H}}=\frac{2 \sigma y_{p}^{2}+y_{p}^{2}+\sigma}{4 \pi y_{p}}$.

The temperature has a minimal value at $y_{p}=1 / \sqrt{3}$ when $\sigma=1$, coinciding with the minimal temperature of a $\mathrm{AdS}_{4}$-Schwarzschild black hole, occurring at $T_{\mathrm{Schw}}=$ $\sqrt{3} /(2 \pi) \approx 0.2757$. Usually, we have two branches of solutions at the same temperature with a fixed value of $\sigma$; one is called large black holes with larger $y_{p}$ and another is small black holes with smaller $y_{p}$.

The boundary conditions are similar to the soliton case. At $x=0$ and $y=0$, the functions $U_{i}$ satisfy the Neumann conditions,

$$
\begin{cases}\partial_{x} U_{i}(0, y)=\partial_{y} U_{i}(x, 0)=0, & i=1,2,3,5, \\ \partial_{x} \tilde{U}_{i}(0, y)=\partial_{y} \tilde{U}_{i}(x, 0)=0, & i=4,6\end{cases}
$$

at $x=1$, we choose $\tilde{U}_{4}=0, U_{3}=U_{5}$, and $\partial_{x} U_{1}=$ $\partial_{x} U_{2}=\partial_{x} U_{3}=\partial_{x} U_{5}=\partial_{x} \tilde{U}_{6}=0$. At $y=1$, we set
$\tilde{U}_{4}=0, \quad \tilde{U}_{6}=\varepsilon\left(1-8 x^{2}+4 x^{4}\right)$ and $U_{1}=U_{2}=U_{3}=$ $U_{5}=1$. Furthermore, we could obtain $U_{1}(x, 0)=$ $U_{2}(x, 0)$ by expanding the equations of motion near $y=0$. The reference metric $\tilde{g}$ is used in the DeTurck method with $U_{1}=U_{2}=U_{3}=U_{5}=1, \quad \tilde{U}_{4}=0$ and $\tilde{U}_{6}=\varepsilon\left(1-8 x^{2}+4 x^{4}\right)$.

We present the typical results of $\tilde{U}_{4}$ and $\tilde{U}_{6}$ with tripolar differential rotation for small and large black hole solutions in Fig. 5. The left column exhibits the distributions of $\tilde{U}_{4}$ for small black hole (top) and large black hole (bottom), and the right column exhibits the distributions of $\tilde{U}_{6}$ for small black hole (top) and large black hole (bottom). We could observe that the deformations of $\tilde{U}_{4}$ and $\tilde{U}_{6}$ for the case of large black hole are larger than the case of small black hole.

\section{A. Entropy}

In this subsection we discuss the entropy of deforming black holes with tripolar differential rotation. The entropy associated with the black hole horizon is given by 

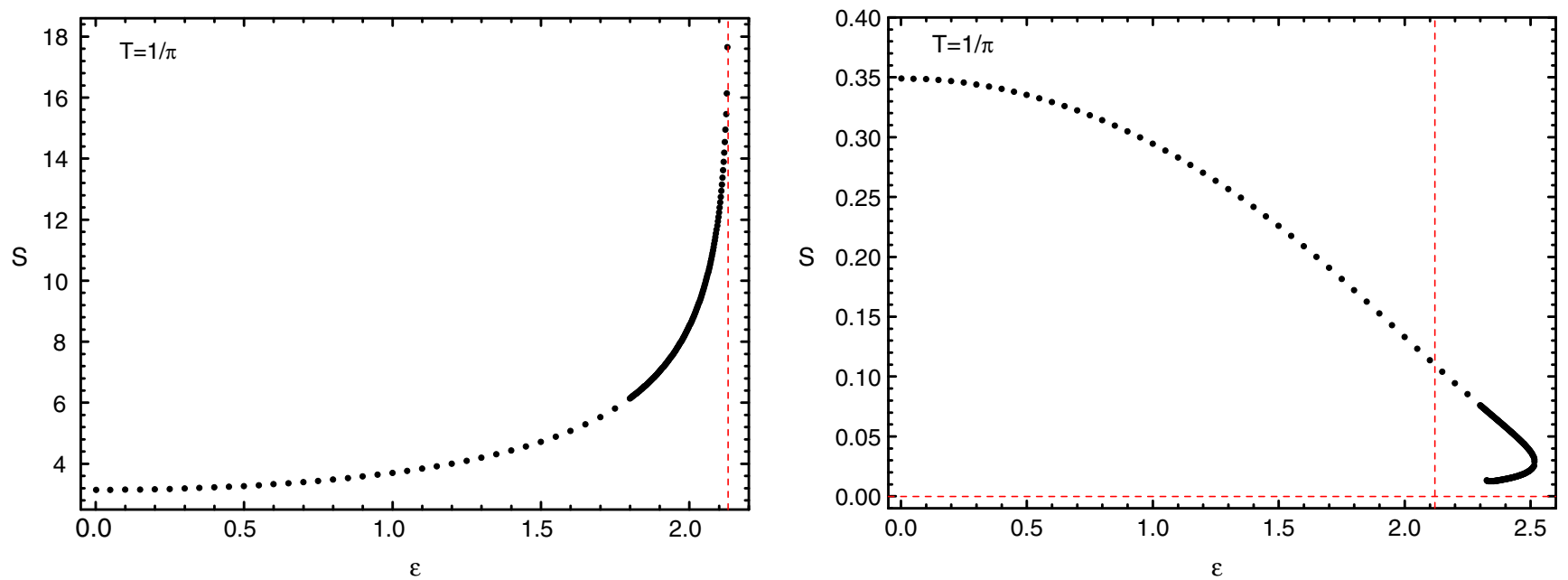

FIG. 6. Left: The entropy against boundary rotation parameter $\varepsilon$ for the large branch of black hole solutions with a temperature $T=1 / \pi$. Right: The entropy against boundary rotation parameter $\varepsilon$ for a small branch of black hole solutions with the same temperature. The vertical red dashed lines in the left and right show $\varepsilon=2.124$ and the horizontal red dashed line shows $S=0$.

$S=\frac{A}{4 G_{N}}=\frac{2 \pi y_{p}^{2} L^{2}}{G_{N}} \int_{0}^{1} d x \frac{1-x^{2}}{\sqrt{2-x^{2}}} \sqrt{U_{3}(x, 0) U_{5}(x, 0)}$.

We present our numerical results of entropy for large and small branches of black hole solutions with temperature $T=1 / \pi$ in Fig. 6, where the vertical red dashed line represents $\varepsilon=2.124$. The entropy for the large branch of black hole solutions with $y_{p}=1$ is shown in the left panel of Fig. 6; we could see that the entropy always increases with the increase of $\varepsilon$, and the axially symmetric black hole solutions with tripolar differential rotation could not be found when $\varepsilon>2.124$. The right panel of Fig. 6 shows the entropy for the small branch of black hole solutions with $y_{p}=1 / 3$. The curve of entropy for a small branch of black hole solutions decreases with the increase of rotation parameter $\varepsilon$, and then it reaches the minimal value at $\varepsilon_{c}=2.5264$. Further decreasing $\varepsilon$, we obtain the second set of solutions with lower entropy and the entropy decreases with the decrease of $\varepsilon$.

We find that in the case of tripolar differential rotation, the norm of Killing vector $\partial_{t}$ in Eq. (2.9) becomes spacelike for the certain regions of $\theta$ with $\varepsilon \in(2,2.124)$. However, solitons and black holes with tripolar differential rotation still exist and do not develop hair due to super-radiance, which is different from the case of dipolar differential rotation. Furthermore, we could obtain the entropy of large black holes at high temperature ( $T \gg T_{\text {schw }}$ ), and we find that the maximum values of $\varepsilon$, below which the stable large black hole solutions could exist, are not a constant under the different high temperatures. With the increase of temperature $T$, the maximum value of $\varepsilon$ decreases, which is different from the case in $[28,32]$. We show our results of entropy for large black holes at different high temperatures in Fig. 7.

In Fig. 7, the lines of different colors represent the curves of entropy of large black holes for different high temperatures with $T \in(1 / \pi, 1.5732)$, and the purple vertical dashed line indicates the maximum value of $\varepsilon$ at $T=1 / \pi$. The inset of Fig. 7 shows the asymptotic behaviors at the maximum values of $\varepsilon$ for $T \in(2 / \pi, 1.5732)$, and the vertical dashed lines of different colors represent the maximum values of $\varepsilon$ at different temperatures. From the inset, it is clear that with the increase of temperature $T$, the maximum value of $\varepsilon$ decreases. It is interesting that when the temperature is much higher than $T_{\text {schw }}$, the maximum value of $\varepsilon$ could be lower than $\varepsilon=2$, which is the critical

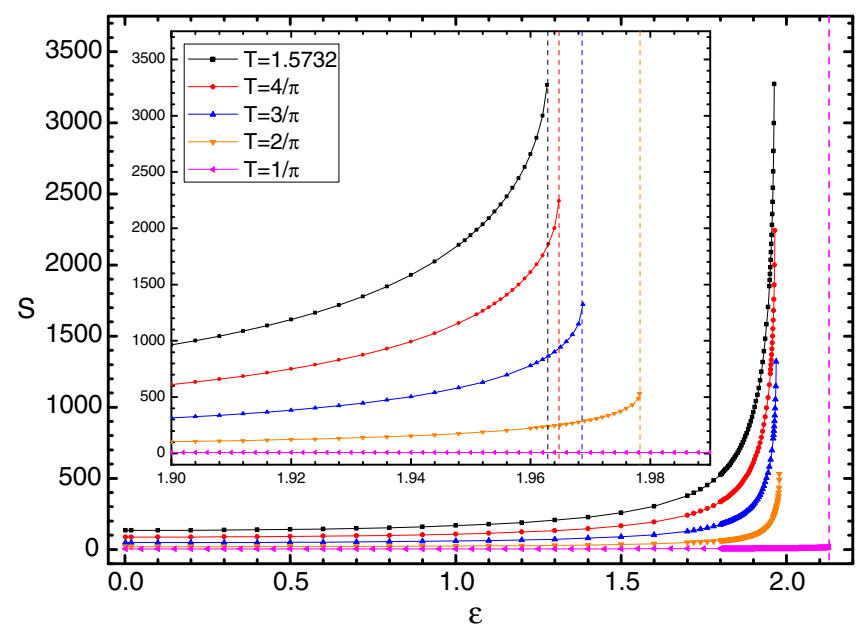

FIG. 7. The entropy against boundary rotation parameter $\varepsilon$ for large black holes at $T \in(1 / \pi, 1.5732)$. The inset shows the asymptotic behaviors of curves of entropy at different temperatures $T \in(2 / \pi, 1.5723)$ around the limits. The vertical dashed lines of different colors represent the limits of different temperatures. The purple line represents the entropy of large black hole at $T=1 / \pi$. Note that because of the scale of the axis, it looks like a straight line. The details of entropy at $T=1 / \pi$ are shown in Fig. 6. 

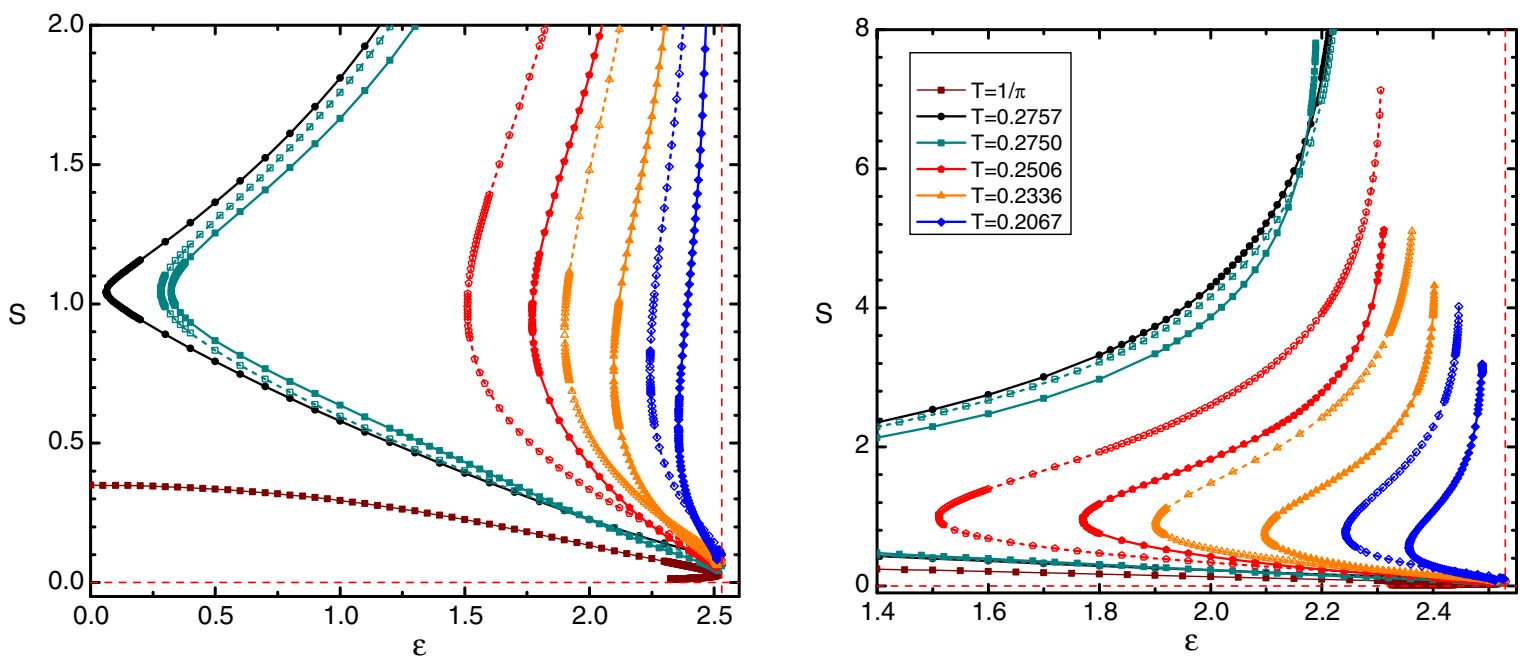

FIG. 8. Left: Entropy against the boundary rotation parameter $\varepsilon$ for low temperature black holes with $T<T_{\text {Schw }}=\sqrt{3} / 2 \pi \simeq 0.2757$. Two types of lines represent large (dashed line with hollow symbols) and small (solid line with solid symbols) black holes, respectively, and the lines of the same color denote the large and small black holes at the same temperature. Right: The asymptotic behaviors for the curves of entropy of large and small black holes. The black solid line represents the black holes with $T \simeq T_{\text {Schw }}$, and the brown solid line represents the small black hole at $T=1 / \pi$. In both panels the vertical red dashed lines indicate $\varepsilon=\varepsilon_{c}$ and the horizontal red dashed lines indicate $S=0$.

value for the Killing vector $\partial_{t}$ becoming spacelike for certain regions of $\theta$. This means that even though the norm of Killing vector $\partial_{t}$ keeps timelike for the some regions of $\varepsilon<2$, the solitons and black holes with tripolar differential rotation could still develop hair due to super-radiance at high temperature.

For the low temperature $T \leq T_{\text {Schw }}=\sqrt{3} / 2 \pi \simeq 0.2757$ situation, we present our results in Fig. 8. The dashed lines with hollow symbols represent the entropy for a large branch of black hole solutions and the solid lines with solid symbols represent the entropy for the small branch of black hole solutions. Two types of lines of the same color denote entropy for large and small branches of black hole solutions, respectively, at the same temperature. For $T>T_{\text {Schw }}$, we first observe that the curves of entropy for two branches of black hole solutions at the fixed temperature are separate (the brown solid line represents the entropy of the small black hole at $T=1 / \pi)$. When temperature is lowered to $T_{\text {Schw }} \simeq 0.2757$, the curves of entropy for two branches of black hole solutions combine into one curve (black solid line). As we continue to lower the temperature to $T<T_{\text {Schw }}$, we again obtain two separate curves of entropy at the fixed temperature, and each curve has two solutions with the fixed value of rotation parameter $\varepsilon$. The solid line with symbols denotes the small black holes, while the dashed line with hollow symbols denotes the large black holes.

\section{B. The horizon geometry}

In order to obtain a better understanding of how the event horizon of the deforming black hole behaves with increasing the boundary rotation parameter $\varepsilon$, we could investigate the geometry of a two-dimensional surface in a curved space by using an isometric embedding in the three-dimensional space [37-41], which has been introduced to study the horizon with dipolar differential rotation embedding in hyperbolic space [28,42]. In the polar coordinates, the metric of hyperbolic three-dimensional space $\mathbb{U}_{3}$ is given by

$d s_{\mathbb{H}^{3}}^{2}=\frac{d R^{2}}{1+R^{2} / \tilde{l}^{2}}+R^{2}\left[\frac{d X^{2}}{1-X^{2}}+\left(1-X^{2}\right) d \phi^{2}\right]$,

where $\tilde{l}$ is the radius of hyperbolic space. The induced metric on the horizon of the black hole with the metric (4.2) is given by

$d s_{\mathrm{IM}}^{2}=L^{2}\left[\frac{4 y_{p}^{2} U_{3}(x, 0)}{2-x^{2}} d x^{2}+y_{p}^{2}\left(1-x^{2}\right)^{2} U_{5}(x, 0) d \phi^{2}\right]$,

and with the pullback of line element (4.6) on the induced metric (4.7), one could obtain an embedding of a twodimensional line element, which is given by a parameter curve $\{R(x), X(x)\}$,

$$
\begin{aligned}
d s_{R X}^{2}= & {\left[\frac{R^{\prime}(x)^{2}}{1+\frac{R(x)^{2}}{\tilde{l}^{2}}}+\frac{R(x)^{2} X^{\prime}(x)^{2}}{1-X(x)^{2}}\right] } \\
& \times d x^{2}+R(x)^{2}\left(1-X(x)^{2}\right) d \phi^{2} .
\end{aligned}
$$

By using Eqs. (4.7) and (4.8) we could obtain a first-order differential equation for the polar coordinate as follows, 


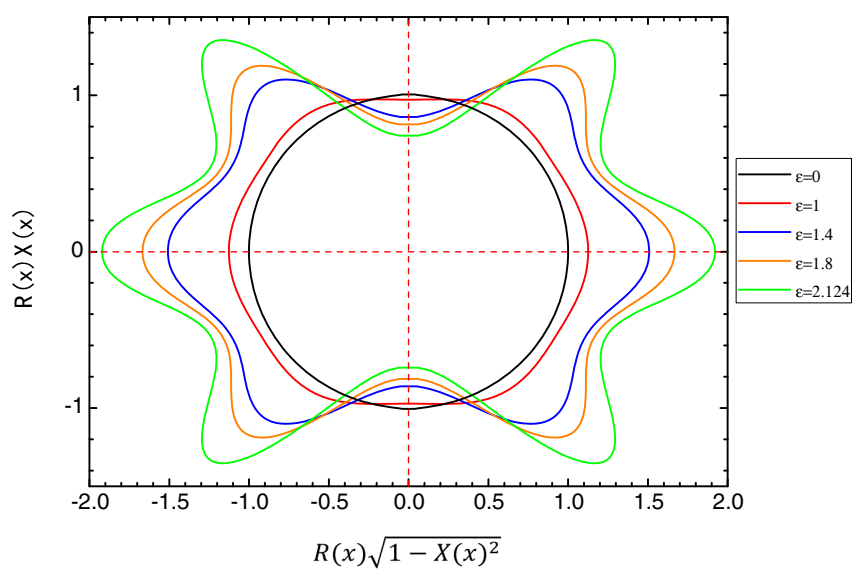

FIG. 9. Hyperbolic embedding of the cross sections of large black hole horizons for several values of the $\varepsilon$ at a fixed temperature $T=13 / 8 \pi$. The black line represents the Schwarzschild-AdS $\mathrm{S}_{4}$ black hole.

$$
\begin{aligned}
& 4 Q(x) D(x)\left(X(x)^{2}-1\right)^{2}\left[D(x)-\tilde{l}^{2}\left(X(x)^{2}-1\right)\right] \\
& \quad+4 \tilde{l}^{2} D(x) X(x)\left(X(x)^{2}-1\right) D^{\prime}(x) X^{\prime}(x)-\left(X(x)^{2}\right. \\
& -1)^{2} \tilde{l}^{2} D^{\prime}(x)^{2}-4 D(x)^{2}\left(\tilde{l}^{2}+D(x)\right) X^{\prime}(x)^{2}=0,
\end{aligned}
$$

with $Q(x)=\left(2-x^{2}\right)^{-1}\left(4 y_{p}^{2} U_{3}(x, 0)\right)$ and $D(x)=y_{p}^{2}\left(1-x^{2}\right)^{2}$ $U_{5}(x, 0)$.

We find it is hard to embed the horizon cross section into hyperbolic space $\mathbb{Q}^{3}$ at the temperature $T=1 / \pi$ with large values of the rotation parameter $\varepsilon$ by using our numerical method, and the deformations of horizon are too small. So we recalculate the embedding of the cross section and obtain the larger deformation of tripolar rotation at $T=13 / 8 \pi$. In Fig. 9, we present the cross sections of the branch of large black hole horizons with tripolar rotation for several values of rotation parameter $\varepsilon$ at fixed temperature $T=13 / 8 \pi$, and we set $\tilde{l}=0.73$ in all plots of

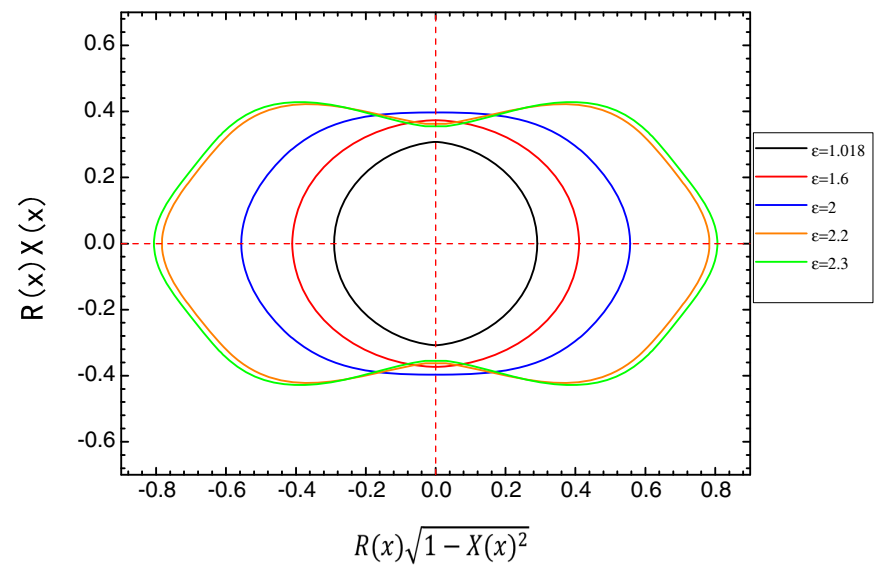

this subsection. As we increase $\varepsilon$, the horizon cross section begins to deform and forms three protrusions, which could extend further at high values of $\varepsilon$.

In Fig. 10, we present the cross sections of horizons at a low temperature $T=0.2606$. In the left panel, we show the branch of large black hole solutions, and we find that the size of the deformation of horizon cross section with tripolar rotation decreases as the temperature decreases. The right panel exhibits the branch of small black hole solutions; we can see that the cross sections of horizons become smaller with the increase of $\varepsilon$. Comparing with the curves of large black holes in the left panel, the curves of small black holes are nearly circular.

\section{The quasinormal modes of black holes}

In this subsection, we discuss the linear stability of the deforming black hole with tripolar differential rotation by studying the quasinormal modes. With the ansatz of the black hole metric (4.2), the scalar field imposed regularity in ingoing Eddington-Finkelstein coordinates $[43,44]$ could be decomposed into

$\Psi(t, x, y, \psi)=e^{i(m \psi-\omega t)} y^{-i \frac{2 \omega y_{p}}{1+3 y_{p}^{2}}}\left(1-y^{2}\right)^{3}\left(1-x^{2}\right)^{|m|} \xi(x, y)$,

where the powers of $x$ and $y$ are chosen to make function $\xi(x, y)$ regular at the origin. The boundary conditions are imposed as follow: at $x= \pm 1$ we require $\partial_{x} \xi(x, y)=0$, and at $y=0$, we require $\partial_{y} \xi(x, y)=0$. At $y=1$, we require

$$
-2 i y_{p} \omega \xi(x, y)+\left(1+3 y_{p}^{2}\right) \partial_{y} \xi(x, y)=0 .
$$

In Fig. 11, for a small black hole, we plot the real part of the quasinormal frequencies $\omega$ as the function of rotation

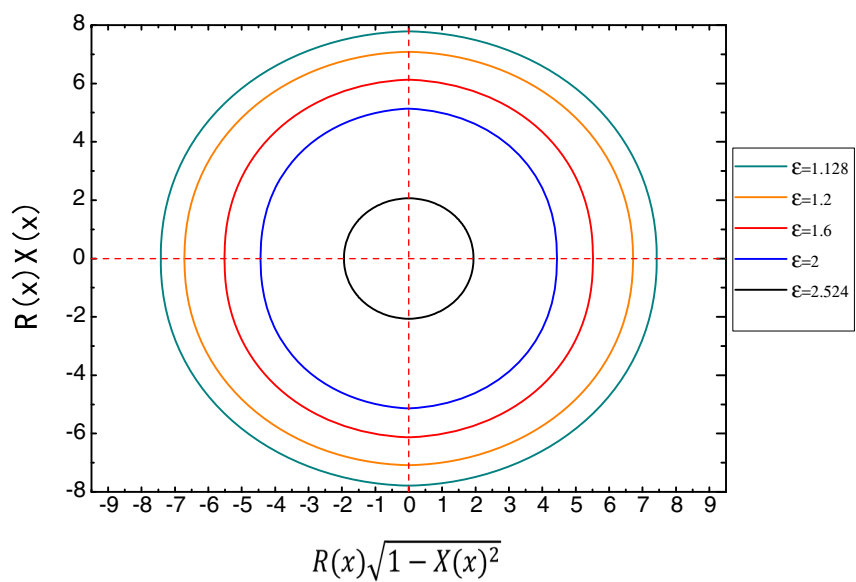

FIG. 10. Hyperbolic embedding of the cross sections of the black hole horizons for several values of $\varepsilon$ at the fixed temperature $T=0.2606$. Left: The embedding for the large branch of black hole solutions with tripolar rotation. Right: The embedding for the small branch of black hole solutions with tripolar rotation. 


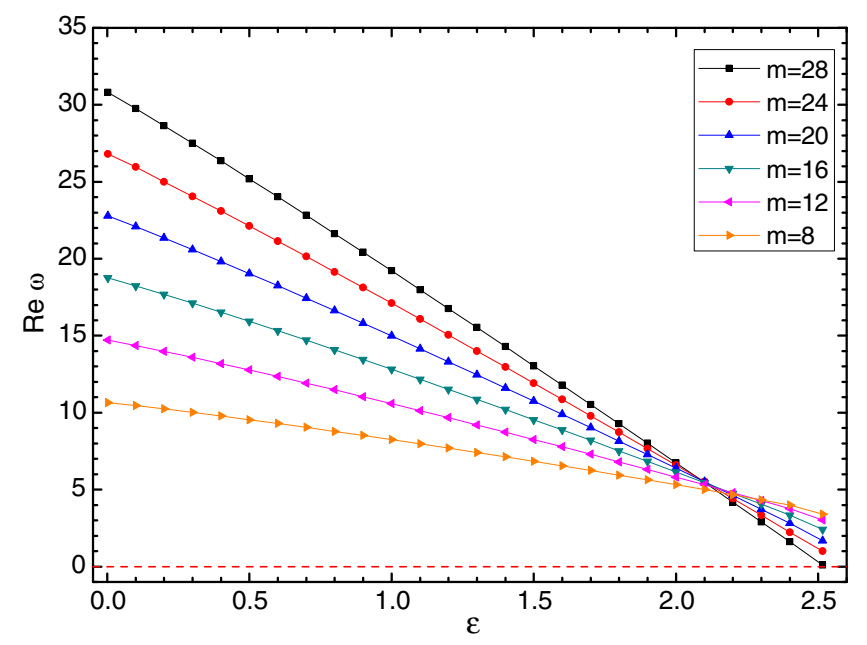

FIG. 11. The real part of frequencies $\omega$ against the rotation parameter $\varepsilon$ for small black hole at the temperature $T=1 / \pi$. The red dashed line indicates $\operatorname{Re} \omega=0$.

parameter $\varepsilon$ for the corresponding values of $m$ at the fixed temperature $T=1 / \pi$, and the red dashed line indicates $\operatorname{Re} \omega=0$. The frequencies $\operatorname{Re} \omega$ with $m \leq 28$ are always positive values in the spectrum of perturbations, and the frequency begins to be negative at a specific value of $\varepsilon$ when $m \geq 29$. The characteristic of $\operatorname{Re} \omega$ against the boundary rotation parameter $\varepsilon$ is similar to that of soliton solutions. Similar to the case of the soliton, for the first unstable mode at $m=29$, one can expect that some branches of black hole with scalar hair $\Psi$ condensation can be found.

\section{CONCLUSIONS}

In this paper, we analyzed the conformal boundary of four-dimensional static asymptotically AdS solutions in Einstein gravity and constructed the numerical solutions of solitons and black holes with odd multipolar differential rotation boundary. Comparing with the dipolar differential rotation solutions in [28], we found that the norm of Killing vector $\partial_{t}$ becomes spacelike for certain regions of $\theta$ with $\varepsilon \in(2,2.124)$; solitons and black holes with tripolar differential rotation do not develop hair due to superradiance at high temperature with $T>T_{\text {Schw }} \simeq 0.2757$, which was different from the case of dipolar rotation. For the large black holes of the high temperature, we found that the maximum value of $\varepsilon$ decreases with the increase of temperature. For $T \in(1.5732,2 / \pi)$, the maximum values of $\varepsilon$ are smaller than 2; meanwhile, the Killing vector $\partial_{t}$ is timelike for some regions of $\varepsilon<2$; solitons and black holes with tripolar differential rotation will develop hair due to super-radiance. When temperature was lowered, we found that the entropy for large and small branches of black holes solutions first combine into one curve at $T=T_{\text {schw }} \simeq$ 0.2757 and then separate into two curves for $T<T_{\text {schw }}$; the entropy for large and small branches of black hole solutions has two solutions at a fixed value of $\varepsilon$. These were different from the results in the cases of dipolar and quadrupolar differential rotation boundaries [28,32]. Furthermore, with the isometric embedding of horizon, it is clearly seen that the black hole horizon is deformed into three protrusions (see Fig. 9). By studying the quasinormal modes, we discussed the linear stability of deforming solitons and black holes with tripolar rotation, respectively, and found that for some branches of solution with scalar hair $\Psi$ condensation, the minimal azimuthal harmonic index $m$ is equal to 24 for solitons and $m=29$ for black holes.

At fixed temperature $T=1 / \pi$, it is interesting to find that though the norm of Killing vector $\partial_{t}$ becomes spacelike for certain regions of $\theta$ with $\varepsilon>2$, solitons and black holes with tripolar differential rotation still exist and do not develop hair due to super-radiance. When temperature is much higher than $T_{\text {schw }}$, we also found that even though the norm of Killing vector $\partial_{t}$ keeps timelike for some regions of $\varepsilon<2$, solitons and black holes with tripolar differential rotation could be unstable and develop hair due to superradiance, which is different from the cases of solutions with dipolar and quadrupolar differential rotation boundaries in $[28,32]$. We checked the numerical solutions with pentapolar differential rotation, which shows very similar results to tripolar differential rotation. There exists the solution of pentapolar differential rotation when $\varepsilon>2$.

At present, we have studied the behaviors of solitons and black holes with tripolar differential rotation, but the angular momentum, energy densities, and thermodynamic properties of black holes with odd multipolar differential rotation have not been studied, and we hope to investigate these in our future work. Besides, we have two extensions of our work. The one extension of our work is to study the action of Einstein-Maxwell gravity in AdS spacetime and construct the deforming charged black holes. We find that there are three branches of solutions because of the existence of charges. The phase diagram of solutions is more complicated than that without charges. The other is to extend the deforming black holes to $f(R)$ gravity and Gauss-Bonnet theory.

\section{ACKNOWLEDGMENTS}

We thank Yu-Xiao Liu, Jie Yang, and Li Zhao for helpful discussion. We also thank the anonymous referees for their valuable comments, which helped to improve the manuscript. Some computations were performed on the shared memory system at the Institute of Computational Physics and Complex Systems in Lanzhou University. This work was supported by the Fundamental Research Funds for the Central Universities (Grant No. lzujbky-2017-182). 
[1] W. Israel, Event horizons in static vacuum space-times, Phys. Rev. 164, 1776 (1967).

[2] R. Ruffini and J. A. Wheeler, Introducing the black hole, Phys. Today 24, 30 (1971).

[3] B. Carter, C. De Witt, and B. S. De Witt, in Proceedings of 1972 Session of Ecole d'Ete De Physique Theorique (Gordon and Breach, New York, 1973).

[4] P. T. Chrusciel, J. Lopes Costa, and M. Heusler, Stationary black holes: Uniqueness and beyond, Living Rev. Relativity 15, 7 (2012).

[5] J. M. Maldacena, The Large N limit of superconformal field theories and supergravity, Int. J. Theor. Phys. 38, 1113 (1999); Adv. Theor. Math. Phys. 2, 231 (1998).

[6] E. Witten, anti-de Sitter space and holography, Adv. Theor. Math. Phys. 2, 253 (1998).

[7] O. Aharony, S. S. Gubser, J. M. Maldacena, H. Ooguri, and Y. Oz, Large $\mathrm{N}$ field theories, string theory and gravity, Phys. Rep. 323, 183 (2000).

[8] Y. Chen, Y. K. Lim, and E. Teo, Deformed hyperbolic black holes, Phys. Rev. D 92, 044058 (2015).

[9] T. Levi-Civita, ds2 einsteiniani in campi newtoniani, Rend. Acc. Lincei 27, 343 (1918).

[10] H. Weyl, Zur Gravitationstheorie, Ann. Phys. (N.Y.) 359, 117 (1917).

[11] J. F. Plebanski and M. Demianski, Rotating, charged, and uniformly accelerating mass in general relativity, Ann. Phys. (N.Y.) 98, 98 (1976).

[12] A. Gnecchi, K. Hristov, D. Klemm, C. Toldo, and O. Vaughan, Rotating black holes in 4d gauged supergravity, J. High Energy Phys. 01 (2014) 127.

[13] D. Klemm, Four-dimensional black holes with unusual horizons, Phys. Rev. D 89, 084007 (2014).

[14] Y. Chen and E. Teo, Black holes with bottle-shaped horizons, Phys. Rev. D 93, 124028 (2016).

[15] Y. B. Zeldovich, Zh. Eksp. Teor. Fiz. Pis. Red. 14, 270 (1971) [Generation of waves by a rotating body, JETP Lett. 14, 180 (1971)].

[16] Y. B. Zeldovich, Zh. Eksp. Teor. Fiz. 62, 2076 (1972) [Amplification of cylindrical electromagnetic waves reflected from a rotating body, Sov. Phys. JETP 35, 1085 (1972)].

[17] W. H. Press and S.A. Teukolsky, Floating orbits, superradiant scattering and the black-hole bomb, Nature (London) 238, 211 (1972).

[18] A. A. Starobinsky, Zh. Eksp. Teor. Fiz. 64, 48 (1973) [Amplification of waves during reflection from a rotating "black hole", Sov. Phys. JETP 37, 28 (1973)].

[19] A. A. Starobinsky and S. M. Churilov, Zh. Eksp. Teor. Fiz. 65, 3 (1974) [Amplification of electromagnetic and gravitational waves scattered by a rotating "black hole", Sov. Phys. JETP 38, 1 (1974)].

[20] S. L. Detweiler and J. R. Ipser, The stability of scalar perturbations of a Kerr-metric black hole, Astrophys. J. 185, 675 (1973).

[21] T. J. M. Zouros and D. M. Eardley, Instabilities of massive scalar perturbations of a rotating black hole, Ann. Phys. (N.Y.) 118, 139 (1979).

[22] S. L. Detweiler, Klein-Gordon equation and rotating black holes, Phys. Rev. D 22, 2323 (1980).
[23] H. K. Kunduri, J. Lucietti, and H. S. Reall, Gravitational perturbations of higher dimensional rotating black holes: Tensor perturbations, Phys. Rev. D 74, 084021 (2006).

[24] Ó. J. C. Dias, G. T. Horowitz, and J. E. Santos, Black holes with only one Killing field, J. High Energy Phys. 07 (2011) 115.

[25] Ó. J. C. Dias and J. E. Santos, Boundary conditions for KerrAdS perturbations, J. High Energy Phys. 10 (2013) 156.

[26] V. Cardoso, Ó. J. C. Dias, G. S. Hartnett, L. Lehner, and J. E. Santos, Holographic thermalization, quasinormal modes and super-radiance in Kerr-AdS, J. High Energy Phys. 04 (2014) 183.

[27] S. R. Green, S. Hollands, A. Ishibashi, and R. M. Wald, super-radiant instabilities of asymptotically anti-de Sitter black holes, Classical Quantum Gravity 33, 125022 (2016).

[28] J. Markeviciute and J. E. Santos, Stirring a black hole, J. High Energy Phys. 02 (2018) 060.

[29] T. Crisford, G. T. Horowitz, and J. E. Santos, Attempts at vacuum counterexamples to cosmic censorship in AdS, J. High Energy Phys. 02 (2019) 092.

[30] G. T. Horowitz, J. E. Santos, and C. Toldo, Deforming black holes in AdS, J. High Energy Phys. 11 (2018) 146.

[31] J. L. Blazquez-Salcedo, J. Kunz, F. Navarro-Lerida, and E. Radu, New black holes in D $=5$ minimal gauged supergravity: Deformed boundaries and frozen horizons, Phys. Rev. D 97, 081502 (2018).

[32] H. B. Li, T. T. Hu, B. S. Song, S. Sun, and Y. Q. Wang, Deforming black holes with even multipolar differential rotation boundary, J. High Energy Phys. 06 (2019) 126.

[33] M. Headrick, S. Kitchen, and T. Wiseman, A new approach to static numerical relativity, and its application to KaluzaKlein black holes, Classical Quantum Gravity 27, 035002 (2010).

[34] T. Wiseman, Numerical construction of static and stationary black holes, in Black Holes in Higher Dimensions (Cambridge University Press, Cambridge, England, 2012), Chap. 10, pp. 233.

[35] Ó. J. C. Dias, J. E. Santos, and B. Way, Numerical methods for finding stationary gravitational solutions, Classical Quantum Gravity 33, 133001 (2016).

[36] V. Cardoso, O. J. C. Dias, G. S. Hartnett, L. Lehner, and J. E. Santos, Holographic thermalization, quasinormal modes and super-radiance in Kerr-AdS, J. High Energy Phys. 04 (2014) 183.

[37] L. Flamm, Beiträge zur Einsteinischen Gravitationtheorie, Phys. Z. 17, 448 (1916) (in particular p. 450) [Republication of: Contributions to Einstein's theory of gravitation, Gen. Relativ. Gravit. 47, 72 (2015)].

[38] L. Smarr, Surface geometry of charged rotating black holes, Phys. Rev. D 7, 289 (1973).

[39] A. Friedman, Isometric embeddings of Riemannian manifolds into Euclidean spaces, Rev. Mod. Phys. 37, 201 (1965).

[40] J. Rosen, Embedding various relativistic Riemannian spaces in pseudo-Euclidean spaces, Rev. Mod. Phys. 37, 204 (1965).

[41] H. Goenner, Local isometric embedding of Riemannian manifolds and Einsteins theory of gravitation, in General Relativity and Gravitation: One Hundred Years after the 
Birth of Einstein, edited by A. Held (Plenum Press, New York, NY, 1980), Vol. 1.

[42] G. W. Gibbons, C. A. R. Herdeiro, and C. Rebelo, Global embedding of the Kerr black hole event horizon into hyperbolic 3-space, Phys. Rev. D 80, 044014 (2009).
[43] G. T. Horowitz and V. E. Hubeny, Quasinormal modes of AdS black holes and the approach to thermal equilibrium, Phys. Rev. D 62, 024027 (2000).

[44] E. Berti, V. Cardoso, and A. O. Starinets, Quasinormal modes of black holes and black branes, Classical Quantum Gravity 26, 163001 (2009). 